\title{
SAVIGNY, HERDER Y LA TENSIÓN ENTRE PARTICULARISMO Y UNIVERSALISMO EN LA CONSTRUCCIÓN DE LA CIENCIA JURÍDICA
}

\author{
SAVIGNY, HERDER AND THE TENSION BETWEEN PARTICULARISM \\ AND UNIVERSALISM IN THE MAKING OF LEGAL SCIENCE
}

\section{DAVID Ricardo LUNA-VinUEZA*}

\begin{abstract}
RESUMEN: Este artículo intenta mostrar la tensión entre particularismo y universalismo en la teoría de Friedrich Karl von Savigny. En otras palabras, se intenta mostrar a Savigny como un autor que se debate entre dos compromisos teóricos diferentes. Para ello, se analiza de forma preliminar la relación entre el pensamiento de Savigny y la filosofía de Johann Gottfried von Herder. La tensión que se propone en este escrito aparece claramente cuando se observa que Savigny se separa de algunas conclusiones del romanticismo de Herder sin abandonar completamente sus tesis fundamentales. Esto hace que el impulso particularista de la teoría sobre el origen del derecho de Savigny se mezcle con la intención universalista del autor cuando construye la noción de ciencia jurídica y la pone en funcionamiento. Incluso, esta tensión se observa en la dogmática de Savigny cuando plantea una diferencia entre el derecho patrimonial y el derecho de familia.
\end{abstract}

Palabras clave: Friedrich Karl von Savigny, Johann Gottfried von Herder, Ciencia Jurídica, Escuela Histórica del Derecho, Romanticismo Alemán.

ABSTRACT: This article attempts to show the tension between particularism and universalism in the theory of Friedrich Karl von Savigny. In other words, it attempts to give an account of Savigny as an author who is rifted between two different theoretical compromises. In this sense, this article starts analyzing the relation between Savigny's thought and Johann Gottfried von Herder's philosophy. The tension proposed in this article appears clearly when it is observed that Savigny rejects certain conclusions of Herder's romanticism, without abandoning his fundamental thesis. This supposes that the particularistic impulse of Savigny's theory on the origin of law mixes with his universalistic intention when he builds the idea of legal science and makes it work. Indeed, this tension can be observed in Savigny's dogmatics when he proposes a difference between patrimonial law and family law.

Key words: Friedrich Karl von Savigny, Johann Gottfried von Herder, Legal Science, Historical School of Law, German Romanticism.

\footnotetext{
* Investigador, Centro de Investigaciones Sociojurídicas (CIJUS), Universidad de los Andes, Bogotá, D.C., Colombia. Correo electrónico: dr.luna129@uniandes.edu.co. Código postal: 111311191. Agradezco a Isabel Cristina Jaramillo, Nicolás Torres, Antonio Barreto Rozo y Daniel Bonilla por sus comentarios a una versión preliminar de este trabajo. Los errores que se puedan encontrar en este artículo se deben únicamente a mis propias falencias intelectuales.
} 


\section{INTRODUCCIÓN}

Varios autores coinciden en que Savigny es un heredero del romanticismo alemán. Así, por ejemplo, Kutner ${ }^{1}, K_{n u d s o n}^{2}$ y Kantorowicz ${ }^{3}$ afirman que varias de las tesis de Savigny están influenciadas de forma considerable por la filosofía de Johann Gottfried von Herder. Por su parte, Engel-Janosi ${ }^{4}$, además de aceptar la influencia de Herder, afirma que otros autores que marcaron una gran influencia sobre Savigny fueron Goethe y Schelling. Estos dos junto con Herder son tres referentes del pensamiento romántico alemán.

Lo interesante de estas menciones es que los autores citados no desarrollan en profundidad la relación entre Savigny y los románticos, sino que se limitan a mencionar este hecho como si se tratara de una verdad aceptada por la comunidad jurídica o un hecho que no merece una explicación detallada. De ese modo, estos autores solo mencionan que los antecedentes más inmediatos de ideas como el "espíritu del pueblo" (llamado también "conciencia popular" o "espíritu nacional") y el organicismo que muestra Savigny al construir su teoría se encuentran en los filósofos románticos ya mencionados. Posiblemente este descuido a los autores que influenciaron el pensamiento de Savigny se deba a que los artículos de Engel-Janosi, Kantorowicz, Knudson y Kutner, así como una buena parte de los artículos en los que se comenta la obra de Savigny, son escritos de divulgación en los que se comunican las ideas de Savigny a la comunidad jurídica 5 .

Este escrito pretende abordar la relación entre el pensamiento de Savigny y el de Johann Gottfried von Herder. Sin embargo, esta investigación tiene, más bien, un fin instrumental. Esta investigación es útil por dos razones. En primer lugar, permite observar con profundidad la relación entre la teoría de Savigny y el ambiente intelectual de su época. Con esto se podrían dar elementos para discutir la opinión de algunos estudiosos como Kantorowicz ${ }^{6}$ que afirman que la teoría de Savigny corresponde enteramente a la tendencia general del pensamiento alemán de su época ${ }^{7}$. En ese sentido, este ejercicio puede ayudar a tener lecturas más contextualizadas, al mismo tiempo que permite abrir la posibilidad de emprender críticas más informadas al pensamiento del autor. En segundo lugar, y esta es la tesis principal de este trabajo, indagar sobre la relación entre Savigny y la filosofía romántica alemana permite ver que este vínculo no es tan pacífico e indudable como lo pretenden mostrar los autores citados más arriba. De hecho, observar hasta qué punto Savigny es un heredero del romanticismo alemán hace más evidente la existencia de una tensión latente

\footnotetext{
KutNer (1972) p. 285.

2 Knudson (2002) p. 410.

3 Kantorowicz (1937) p. 385.

4 Engel-Janosi (1947) p. 707.

5 Este tipo de artículos son muy comunes en inglés, tal y como lo muestra la bibliografía al final de este escrito.

6 Kantorowicz (1937) p. 336.

7 En cierta forma, los artículos de Dufour (1981) y Zuleta (1977) intentan contradecir una opinión como la de Kantorowicz. En términos generales, ambos autores ven una ruptura de Savigny con respecto a la filosofía romántica. El enfoque de este artículo es distinto al de Dufour y de Zuleta en tanto quiere enfatizar una tensión en el pensamiento del Savigny en lugar de una ruptura.
} 
entre universalismo y particularismo su teoría ${ }^{8}$. Esto, por tanto permitiría relajar un poco la opinión de Kantorowicz al mostrar elementos distintivos del pensamiento de Savigny en relación con su época. Esta tensión ha sido planteada por Kennedy ${ }^{9}$ al estudiar la forma en la que Savigny sistematiza el derecho privado. En este escrito se quiere mostrar que esta tensión puede ampliarse a otras esferas del pensamiento de Savigny.

Con esto en mente este escrito se divide en tres partes. En la primera de ellas se exponen la teoría general sobre el origen y desarrollo del derecho de Savigny y la teoría sobre el origen del lenguaje de Herder. Este ejercicio se realiza porque Savigny parece referirse de forma más o menos explícita a la teoría del lenguaje de Herder como el antecedente más inmediato de su pensamiento sobre el derecho. Posteriormente, se encontrarán los puntos en común entre estas dos teorías y se mostrará que entender el pensamiento de Herder arroja elementos necesarios para una mejor comprensión de la teoría de Savigny, en especial con respecto al carácter normativo o descriptivo de algunas de sus tesis. En la segunda parte del texto se mostrará que a pesar de los puntos en común entre Savigny y Herder existen otros elementos importantes de la teoría del primero que no tienen correspondencia en la filosofía de Herder y que, incluso, pueden llegar a contradecirla. Esta separación entre Savigny y Herder abre las puertas para preguntase sobre la tensión entre particularismo y universalismo en Savigny, especialmente en lo que se refiere a la ciencia del derecho, la teoría de la interpretación y las categorías en las que separa el derecho privado. Por último, se aportan algunas conclusiones.

\section{LA TEORÍA SOBRE EL ORIGEN DEL DERECHO DE SAVIGNY Y LA TEORÍA SOBRE EL ORIGEN DEL LENGUAJE DE HERDER}

\subsection{Las Características de la teoría SOBRE El ORIGEN DEL DERECHO De SAVIGNY}

Como lo muestra Reimann ${ }^{10}$, Savigny aprovechó el debate alrededor de la codificación alemana para exponer por primera vez su teoría general sobre el origen del derecho ${ }^{11}$. En esa oportunidad Savigny reaccionó contra la propuesta de codificación de Anton Friedrich Justus Thibaut. Según Thibaut el derecho alemán debería ser codificado como el derecho francés, el austriaco y el prusiano como una señal de la unidad del pueblo alemán ${ }^{12}$.

\footnotetext{
8 Cfr. Fernández-Crehuet (2008) pp. 160-165. El autor sugiere esta tensión, pero le da un tratamiento distinto al de este trabajo.

9 Kennedy (2010).

10 Reimann (1989) p. 98 Reimann ( (1990) p. 851

11 Reimann afirma que la teoría general del derecho de Savigny se encuentra principalmente en cuatro textos [Reimann ( (1990) p. 852 nota 61]: Metodología Jurídica escrito entre 1802 y 1803 [SAVIGNy (2004)], De la vocación de nuestro siglo para la legislación y la ciencia del derecho escrito en 1814 [SAVIGNY (1977)], el Sistema de derecho romano actual [cuyos parágrafos pertinentes se encuentran en [SAVIGNY (1949)] y su ensayo introductorio a la Revista para la ciencia histórica del derecho (Zeitschrift für geschichtliche Rechtswissenschaft) de 1815. Esta opinión es compartida por Mollnau, excepto por la mención a Metodología Jurídica [Mollnau (1989) p. 84].

12 Kantorowicz (1937) p. 332. No hay que olvidar que esta controversia se da en medio de la discusión sobre la formación de los Estados nacionales. Como se verá a lo largo de este escrito, el nacionalismo es un elemento que hay que tener en cuenta para entender en pensamiento de Savigny y su relación con el romanticismo. Agradezco a Antonio Barreto Rozo por hacerme notar esto.
} 
Esta propuesta tenía en mente la profunda dispersión geográfica del derecho alemán y la complejidad del derecho privado romano que se encontraba vigente aún en ausencia de un derecho verdaderamente propio ${ }^{13}$. De acuerdo con Kutner, la propuesta de Thibaut mostraba tanto un nacionalismo exacerbado por la derrota del proyecto imperial de Napoleón como una gran desconfianza a la apropiación del derecho romano u otro derecho extranjero ${ }^{14}$.

El proyecto de Thibaut, sin embargo, podía ser pensado como una rara mezcla entre el derecho natural y las teorías sobre el derecho positivo. Savigny identificó claramente estos rasgos de la propuesta al observar que más que una lucha por la organización de la justicia civil, lo que realmente estaba detrás de la propuesta de Thibaut era un nacionalismo mal entendido ${ }^{15}$. En ese sentido, para Savigny el proyecto codificador de Thibaut tenía dos premisas fundamentales. En primer lugar, el deseo de organización del derecho alemán suponía un olvido de todo cuanto "había de característico y de grande en los demás siglos, al par que por el natural desenvolvimiento de los pueblos y de las instituciones, es decir, de todo aquello que la historia produce de más saludable y provechoso, fijando exageradamente la atención en la época actual, que se creía destinada nada menos que a la efectiva realización de la perfección absoluta"16.

Esto quiere decir que este afán por la organización del derecho implicaba aceptar que el código debía ser un proyecto racional aplicable a todos los pueblos y a todos los tiempos sin tener en cuenta la influencia histórica de cada nación ${ }^{17}$. En ese sentido, Savigny identificó que si bien Thibaut era un nacionalista, su proyecto estaba íntimamente relacionado con la perspectiva universalizante del derecho natural ${ }^{18}$. En segundo lugar, de acuerdo con Savigny, Thibaut tenía una opinión general sobre el origen del derecho positivo. Savigny explica que Thibaut aceptaba como premisa de su propuesta que "todo derecho en su estado normal no es más que el resultado de la ley, esto es, de los actos emanados de la potestad suprema del Estado"19. Esto implicaba para Savigny que aceptar la tesis de Thibaut llevaría a que la legislación y el contenido de la ciencia jurídica fueran siempre accidentales y contingentes ${ }^{20}$. Si bien Savigny no lo plantea de ese modo, su reflexión muestra que la propuesta de Thibaut sufre de una contradicción fundamental: por un lado, enfatiza el proyecto racional y universalizante del derecho natural, pero, por otro, somete el contenido del derecho a la contingencia al dejarlo en manos del legislador.

El punto de partida de Savigny muestra que su teoría del derecho se construye como una oposición a las dos premisas de Thibaut. El primer paso de la crítica se enfoca hacia el método del derecho natural21 ${ }^{21}$ Como lo muestra Reimann, el desarrollo de la ciencia jurí-

\footnotetext{
13 Reimann (1989) p. 97.

14 Kutner (1972) p. 282.

15 SaVigny (1977) p. 37.

16 SAVIGNy (1977) pp. 39-40.

17 SaVigny (1977) p. 40.

18 SaVigny (1977) p. 42.

19 SAVIGnY (1977) p. 41

20 Dufour (1981) p. 22.

21 Para una explicación de la noción de derecho natural que no enfatiza su método, véase GómEz (1999) pp. 254-261.
} 
dica alemana entre los siglos XVII y XVIII tuvo como base una crítica del método del derecho natural. Específicamente, Reimann afirma que el concepto mismo de ciencia jurídica surgió como una necesidad de pensar en una nueva metodología para abordar el derecho después de que la filosofía crítica de Kant destruyera la fe en las premisas y métodos del derecho natural ${ }^{22}$. La metodología del derecho natural suponía la existencia de principios fundamentales del derecho inherentes a la naturaleza humana, $y$, en cuanto tales, con una validez más allá de cualquier contexto histórico. En ese sentido, para crear un sistema estrictamente lógico y comprensivo de derecho natural las reglas se deducirían de forma matemática de los principios fundamentales ${ }^{23}$. Para Reimann esta es la característica principal del método de Christian Wolff, el principal exponente del derecho natural en Alemania ${ }^{24}$. Lo problemático del método del derecho natural no era el uso de la razón en cuanto tal, sino la mezcla entre ética y derecho, entre un enfoque normativo y uno descriptivo para construir los principios que servirían para deducir el sistema de derecho natural. En ese sentido, la crítica de Kant se enfocaba en mostrar que en los sistemas de derecho natural las reglas positivas y los estándares ideales no se distinguían correctamente. Así, al mezclarse elementos normativos con elementos descriptivos el resultado era un esquema con un escaso valor analítico. De ese modo, la propuesta era separar la filosofía del derecho, la cual se encargaría de las preguntas de tipo normativo, de la ciencia del derecho, la cual se basaría en la observación y la experiencia ${ }^{25}$.

Savigny se adhiere a esta crítica al afirmar que el proyecto codificador supone siempre que existe un derecho ideal al cual debe tender el derecho positivo. El código como proyecto racional no traduce el pensamiento del pueblo porque representa una imposición de un derecho que debe ser como un ordenamiento positivo. Es decir, para unificar el derecho propiamente alemán, lo cual sería coherente con el nacionalismo de Thibaut, se usa lo que el derecho debe ser desde una perspectiva racional. De ahí que Savigny encuentre que el proyecto codificador olvida la influencia histórica en el desenvolvimiento de cada pueblo. Así, para hacer eco de la división entre ciencia del derecho y filosofía del derecho de la que se habló antes, Savigny se dedicará en mayor medida a describir y sistematizar el derecho que observa en la realidad.

La reacción frente a la segunda premisa de Thibaut es, tal vez, el enunciado más famoso de la teoría de Savigny. Frente a pensar que el origen del derecho positivo se encuentra en la ley, Savigny afirma que "el derecho vive al igual que la lengua en la conciencia popular" ${ }^{26}$. La ley, entonces, solo es la expresión visible del derecho, y, en cuanto tal, es "un pronunciamiento del poder supremo del Estado sobre la regla jurídica" ${ }^{27}$. De ese modo, Savigny afirma que "el sujeto en el que y para el que el derecho positivo tiene su existencia

22 Reimann (1990) pp. 892-893.

23 Sobre este punto véase Zuleta (1977) p. 64.

24 Es interesante notar que los primeros contactos de Savigny con la teoría del derecho se dieron con los libros de Wolff. Cfr. Kutner (1972) p. 280.

25 Reimann (1990) p. 845.

26 SaVigny (1949) p. 35. Savigny (1977) p. 44.

27 SAVIGNY (1949) p. 31. 
lo encontramos en el pueblo"28 (énfasis fuera del texto), de tal forma que es la conciencia del pueblo y no la voluntad de cada individuo o una asamblea legislativa la que produce el derecho positivo ${ }^{29}$.

Para Savigny esto se puede comprobar de dos formas claramente observables en la realidad. En primer lugar, cada pueblo reconoce de forma general y uniforme su derecho positivo $^{30}$. Esto hace que el derecho mismo haga parte del conocimiento popular ${ }^{31}$ por la conciencia que cada pueblo tiene de su ser. En segundo lugar, Savigny piensa que el derecho es un fenómeno similar al lenguaje, el cual es una particularidad de cada pueblo ${ }^{32}$. Así, al contemplar las instituciones jurídicas en un contexto orgánico se llega a la conclusión de que expresa al igual que el lenguaje la naturaleza individual de cada pueblo. En palabras de Engel-Janosi, para Savigny tanto el lenguaje como el derecho expresan la conciencia del pueblo o la conciencia común nacional; es decir, tanto el lenguaje como el derecho no tienen una existencia aislada, sino que son aspectos particulares de la vida de cada pueblo ${ }^{33}$.

Esta forma de pensar el origen del derecho tiene dos implicaciones. Por un lado, si existe una verdadera analogía entre el derecho y el lenguaje debe haber un mecanismo que le dé estabilidad al derecho positivo; en otros términos, debe haber una forma en la que el derecho positivo sea usado y respetado de forma ineludible como las reglas del lenguaje. Para Savigny, mientras la estabilidad en el lenguaje se da por su uso constante e ininterrumpido, lo que mantiene al derecho de una forma determinada son ciertos "actos simbólicos, en los cuales, los principios del derecho toman vigor y fuerza, cuando no resultan absorbidos y dominados" 34 . Estos actos simbólicos son expresiones formales que muestran las costumbres reconocidas por cada pueblo determinado. En ese sentido, el derecho depende de la costumbre en tanto esta es la forma en la que se revela la conciencia de cada pueblo ${ }^{35}$. Dicho de otra forma, la costumbre es el síntoma del derecho positivo ${ }^{36}$. De ese modo, Savigny justifica el derecho como la revelación a través de la costumbre de la conciencia del pueblo ${ }^{37}$ en tanto la costumbre es el signo externo a través del cual esta se muestra ${ }^{38}$. Por otro lado, de la misma forma que sucede con el lenguaje, el progreso del derecho y su transformación debe obedecer a una ley de intrínseca necesidad ${ }^{39}$. Es decir, el derecho depende de la expresión particular de la conciencia de cada pueblo en las etapas de su desarrollo. Esto implica que el derecho se desarrolla de forma orgánica en "virtud de una fuerza

\footnotetext{
28 SAVIGny (1949) p. 35.

29 Cfr. Dufour (1981) p. 21. El autor afirma que este rasgo de la teoría de Savigny se puede entender como una parte de su crítica al pensamiento ilustrado.

30 SaVignY (1949) p. 36

31 SAVIGNY (1977) p. 44.

32 Savigny (1949) p. 36.

33 Engel-Janosi (1947) p. 52.

34 SaVigny (1977) p. 45.

35 SAVIGNY (1977) p. 46

36 Savigny (1949) P. 51.

37 FrankLIN (1970) p. 776.

38 FrankLin (1959) p. 70.

39 SAVigny (1949) p. 37. Savigny (1977) p. 46.
} 
íntima y tácitamente activa y no en virtud de la arbitrariedad de un legislador" ${ }^{30}$. Como lo indica Kantorowicz, esa idea supone que el derecho no solo se desarrolla, sino que también se crea en virtud de factores naturales y sociales y no como la expresión de la arbitrarie$\operatorname{dad}^{41}$. En ese sentido, el derecho es pensado como un fenómeno sujeto a una evolución constante y orgánica ${ }^{42}$ y que solo puede ser entendido al tener en cuenta la dimensión histórica de cada pueblo particular ${ }^{43}$.

Ahora bien, el pueblo al que se refiere Savigny tiene una connotación muy específica, al igual que la noción de Estado que se deriva de ella. Para Savigny el pueblo es una "comunidad espiritual que en el uso del mismo lenguaje se evidencia, robustece y desarrolla" ${ }^{\text {" }}$. El pueblo es visto por el autor como un organismo colectivo que posee una individualidad propia $^{45}$. El pueblo, en este punto, es una unidad invisible y abstracta que se manifiesta por medio del uso de un lenguaje común. Esta referencia al lenguaje como elemento definitorio del pueblo será muy importante en el desarrollo de este escrito. Con esta definición en mente Savigny establece que el pueblo adquiere una apariencia visible en el Estado: "el Estado es el cuerpo de la comunidad espiritual del pueblo y con él tenemos a la vez fronteras rigurosamente determinadas de la unidad" ${ }^{2}$. El Estado es la expresión jurídica del pueblo, la cual, como todo el derecho, adquiere una forma específica para él: "Esta afirmación no se refiere solo a la existencia de un Estado en general, sino también a la forma particular que el Estado en cada pueblo reviste, puesto que también la producción del Estado es una clase de producción de derecho, inclusive la suprema forma de toda producción de derecho" ${ }^{47}$.

Es importante enfatizar que lo que se ha escrito hasta ahora corresponde al origen del derecho positivo. Este énfasis no es gratuito, sino que permite ver, a su vez, dos aspectos interesantes de la teoría de Savigny: por un lado, una dimensión de su crítica al origen racional del derecho; $y$, por otro, la magnitud de un paso analítico del autor para la teoría general.

Franklin muestra que la concepción de la conciencia popular desarrollada por Savigny tiene como aspecto principal la superación de la idea rousseauniana de la hegemonía de la voluntad general ${ }^{48}$. La hegemonía de la voluntad general en la creación del derecho y del Estado mismo tiene su base en la conciencia racional de seres humanos que se determinan a sí mismos. Según Franklin esta construcción tiene como punto de partida el derecho natural racional. Savigny, en cambio, al situar el origen del derecho positivo en la

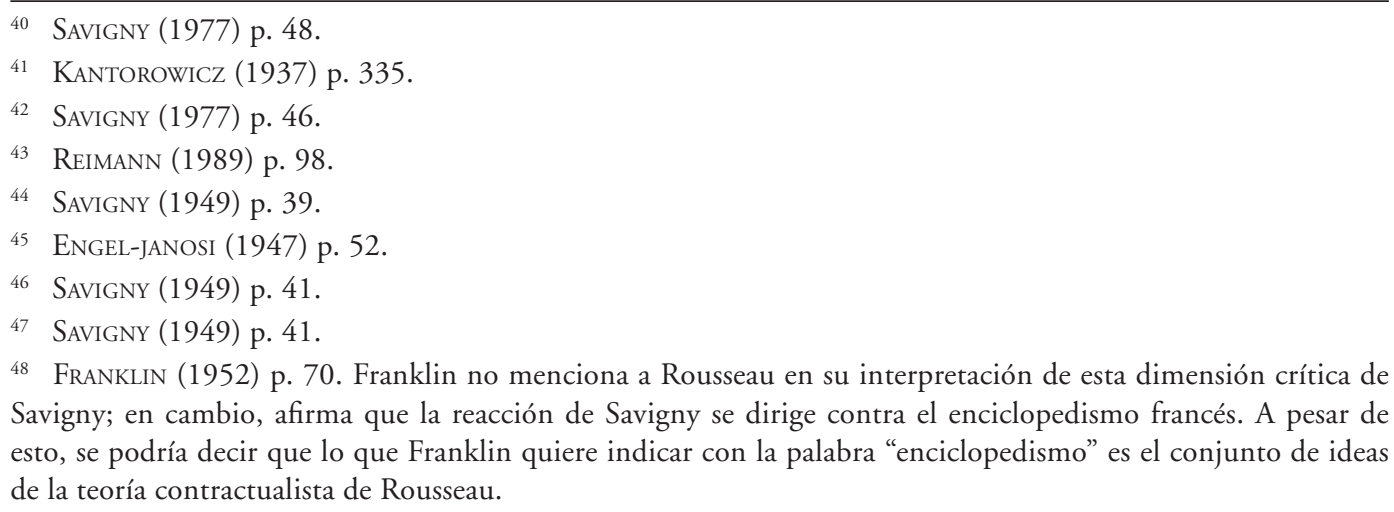

48 FrankLIN (1952) p. 70. Franklin no menciona a Rousseau en su interpretación de esta dimensión crítica de Savigny; en cambio, afirma que la reacción de Savigny se dirige contra el enciclopedismo francés. A pesar de esto, se podría decir que lo que Franklin quiere indicar con la palabra "enciclopedismo" es el conjunto de ideas de la teoría contractualista de Rousseau. 
conciencia popular, lo ubica en una conciencia orgánica y organizada que no se agota en la relación entre las conciencias individuales. Si bien Franklin no lo afirma, se podría decir a partir de él que Savigny reinterpreta la noción de voluntad general. Savigny establece que "el derecho existe en el espíritu común del pueblo [...] o sea en la voluntad común que por ello lo es de cada individuo" ${ }^{49}$. Esta voluntad común no tiene un sustento racional como la voluntad contractualista, sino que es esencialmente irracional en tanto su expresión son fuerzas invisibles como la costumbre, que si bien el individuo no puede controlar, sí puede percibir su influencia sobre sí mismo. En ese sentido es claro que la voluntad común de Savigny controla a los individuos sujetos al derecho porque los atraviesa sin que lo perciban, a diferencia de la teoría contractualista en la que la voluntad depende en última instancia de los sujetos políticos ${ }^{50}$. En ese sentido es claro que, para Savigny, la conciencia popular existe fuera de los individuos y no puede aprehenderse por medio del conocimiento racional ${ }^{51}$. De ese modo, lo único que puede observarse son sus signos externos como la costumbre y el lenguaje.

Por otro lado, como se dijo, enfatizar que lo anterior se refiere al origen del derecho positivo permite ver la magnitud del siguiente paso analítico de Savigny. El autor es claro al afirmar que su argumento sobre el origen se tiene que entender en un estado primitivo de la humanidad: "Preguntaremos ante todo a la historia cómo se ha desenvuelto realmente el derecho entre los pueblos primitivos con el fin de procurar ver y juzgar qué es lo que hay en ese desenvolvimiento de necesario, de útil o de censurable [...]. Podemos prescindir desde luego de tal doctrina [que indica que la infancia de la sociedad se ha pasado en una condición perfectamente animal] y limitarnos al hecho de este primer estado en que indudablemente se ha encontrado el derecho civil [...]. Aquella clara y natural condición manifiestase principalmente en el derecho civil" 52 .

Esto es importante para entender el cambio tan grande que introduce Savigny en su teoría al afirmar que en los estados civilizados de la humanidad y como consecuencia de la especialización de labores "lo que antes vivía en la conciencia popular, conviértese en adelante en materia de la competencia de los juristas, que en tal concepto vienen a representar al pueblo" 53 . De ese modo, si bien el derecho no deja de vivir en la conciencia del pueblo, por lo que siempre conserva una dimensión que Savigny llama "política", ahora es un objeto de elaboración científica. Esto quiere decir que el derecho tiene en los estados avanzados de la civilización una vida separada y científica como elemento "técnico" 54 . Este paso analítico de la teoría general del derecho de Savigny es desconcertante: si bien se puede aceptar el argumento de la especialización del trabajo jurídico, no parece muy claro por qué el derecho requiere de elaboración científica o por qué la forma de acceder a él es ahora científica. Este hecho es aún más perturbador si se tiene en cuenta que Savigny afirma que "las

\footnotetext{
9 Savigny (1949) p. 42.

Franklin (1952) p. 70.

51 Franklin (1952) p. 70.

32 Savigny (1977) pp. 43-44.

53 Savigny (1977) p. 47.

54 SAVIGNY (1977) p. 47.
} 
normas son establecidas por la ciencia del derecho" 55 . A primera vista pareciera que el autor deja de lado su teoría sobre el origen basada en la conciencia popular para concentrarse en pensar en una aproximación científica al derecho.

Ahora bien, las referencias al origen y desarrollo del lenguaje que hace Savigny para explicar su teoría sobre el origen del derecho son muy numerosas. En especial, aquella en la que Savigny afirma que el derecho se puede pensar de forma analógica al lenguaje en tanto ambos tienen un nacimiento invisible y trascendente en los pueblos no deja de causar sorpresas. Precisamente, son estas referencias las que hacen pensar a Kutner ${ }^{56}$, Knudson ${ }^{57}$, Kantorowicz ${ }^{58}$ y Engel-Janosi ${ }^{59}$ que existe una relación muy fuerte entre la teoría de Savigny y la filosofía de Herder. La magnitud de esta relación, como se dijo en la introducción, no es abordada por los autores citados. Por esa razón, en lo que sigue se expondrán las teorías sobre el origen del lenguaje y sobre la organización política de Herder con el fin de buscar las similitudes con la teoría general del derecho de Savigny.

\subsection{La teoría Sobre el origen del lenguaje de Herder}

La teoría sobre el origen del lenguaje de Herder está recogida en su Ensayo sobre el origen del lenguaje $\mathrm{e}^{60}$ escrito en 1770 y en el capítulo II del libro IX de sus Ideas para una filosofía de la historia de la humanidad ${ }^{61}$, escritas entre 1784 y 1791 . Algunas de las conclusiones de su teoría sobre el origen del lenguaje se trasladan a su forma de pensar la organización política. Para explicar el origen del lenguaje Herder construye un argumento en cuatro partes, cada una de las cuales está antecedida de una proposición que el autor denomina "ley de la naturaleza" 62 .

La primera de las leyes de la naturaleza afirma que "el ser humano es un ser libre, pensante y creativo, cuyas facultades operan en un progreso continuo: por lo tanto, está predispuesto al lenguaje" ${ }^{63}$. Herder parte del hecho de que tanto los seres humanos como lo animales pueden expresar sus sentimientos por medio de sonidos particulares. Esta facultad es llamada por el autor "lenguaje original" ${ }^{4}$. Para Herder, sin embargo, esta no constituye

55 SAVIgny (2004) p. 19.

56 Kutner (1972) p. 285.

57 KNUDSON (2002) p. 410.

58 Kantorowicz (1937) p. 385.

59 ENGEL-JANOSi (1947) p. 52.

60 Herder (2010a).

61 Herder (2010b).

62 El Ensayo sobre el origen del lenguaje está dividido en dos partes. La primera titulada "¿Era posible para los seres humanos inventar el lenguaje solamente a partir de sus propias capacidades naturales?” ("Was it possible for man to invent language solely by his own natural facilities?”) es una crítica a las teorías de Candillac, Rousseau y Süssmilch quienes explicaban el origen del lenguaje a partir de la animalidad del ser humano. En la segunda parte titulada "De cómo los seres humanos pudieron y debieron haber inventado el lenguaje de la forma más apropiada" ("How man could and must have invented language in the most appropriate manner") Herder expone su teoría haciendo uso de las leyes de la naturaleza. Esta exposición se enfocará en la segunda parte.

63 Herder (2010a) p. 153. "Man is a free, thinking, and creative being whose faculties operate in a continuous progression: hence he is predisposed for language".

64 Herder (2010a) p. 119. 
una buena explicación del "discurso humano" en la medida en que el lenguaje propiamente humano parece ser aquel en el que el individuo es consciente de cada uno de los sonidos que emite ${ }^{65}$. Para llegar a esta conclusión Herder afirma que lo que distingue a los seres humanos de los animales es la existencia de una mente reflexiva. Esto es así porque ante la ausencia de instintos la naturaleza ha hecho del ser humano no solo capaz de conocer, desear y actuar, sino también consciente de que conoce, desea y actúa ${ }^{66}$. Esta conciencia convierte al ser humano en un ser reflexivo en la medida en que es capaz de distinguir tipos de sensaciones, concentrarse en imágenes determinadas, aislar sus características y hallar relaciones entre cada sensación. Para Herder esta capacidad natural de apercepción ${ }^{67}$ del ser humano supone la existencia de un juicio en su mente. Este juicio solo es posible por la palabra. En ese sentido, el lenguaje siempre está latente como una característica natural del ser humano en tanto ser reflexivo ${ }^{68}$. En otros términos, la posibilidad misma de la conciencia no hubiera sido posible sin la habilidad natural del ser humano de organizar cadenas de pensamiento a través de cadenas de palabras ${ }^{69}$.

La segunda de las leyes de la naturaleza afirma que "el ser humano es por naturaleza una criatura gregaria, nacida para vivir en sociedad: por lo tanto, el desarrollo del lenguaje es tanto natural como esencial para él"70. Herder desarrolla este argumento apelando a la noción de familia. De acuerdo con el autor, los seres humanos nacemos siempre en familias, las cuales nos proveen de protección en la medida en que somos arrojados al mundo con una debilidad absoluta y desprovista de instintos. Sin embargo, esta característica del ser humano hace que sea necesario que reciba una instrucción para superar sus falencias naturales. Quienes instruyen a los seres humanos son sus padres y el objeto de la instrucción es el legado espiritual de cada familia. Para transmitir este legado son necesarios el desarrollo y la intermediación del lenguaje ${ }^{71}$. De allí que el lenguaje sea también esencial para el ser humano. Por lo anterior, el lenguaje, que en la primera ley de la naturaleza parecía tener una dimensión puramente individual en tanto elemento que permite la reflexión, adquiere dos características importantes. En primer lugar, el lenguaje es el medio a través del cual son posibles las relaciones con los otros ${ }^{72}$. En segundo lugar, el lenguaje lleva consigo la herencia de cada familia y es el medio a través del cual se perpetúa su forma de pensar ${ }^{73}$. El lenguaje marca la pertenencia a una familia y hace evidente que cada ser humano hace parte de una cadena de generaciones y del progreso de toda la especie. Estas características hacen pensar a Barnard que el lenguaje permite la diferenciación entre un Yo y un Otro: dado

\footnotetext{
65 Herder (2010a) pp. 124-125.

66 Herder (2010a) p. 132.

67 De acuerdo con la 22a edición del diccionario de la Real Academia Española de la Lengua, "apercepción” significa "percepción atenta y clara, con conciencia de ella".

68 Herder (2010a) p. 135.

69 Herder (2010a) p. 157.

70 Herder (2010a) p. 161. "Man is by nature a gregarious creature, born to live in society; hence the development of language is both natural and essential for him".

71 Herder (2010a) p. 163.

72 Barnard (2010) p. 22.

73 Herder (2010a) p. 163.
} 
que el lenguaje es el medio a través del cual el ser humano adquiere conciencia de su yo por medio de la reflexión, también es el medio que le permite distinguirse de los demás ${ }^{74}$.

La tercera ley de la naturaleza tiene una íntima relación con la anterior: "dado que la raza humana no es un grupo homogéneo, no habla el mismo lenguaje. La formación de las diversas lenguas nacionales es, por tanto, el corolario natural de la diversidad humana"75. Herder explica que las familias conformaron los pueblos de tal manera que crearon un nuevo legado popular a partir de los suyos. Las familias que conformaron cada pueblo ahora participan del carácter específico de la nación ${ }^{76}$. Cada pueblo se caracteriza por ser un todo orgánico en virtud de las relaciones entre sus miembros. El pueblo no es una cosa que tenga una existencia aislada de sus miembros, sino que es un "hecho relacional un continuo histórico y cultural"77. Como sucedía con las familias, los pueblos empiezan a crear y a transmitir su legado interno compuesto por el conjunto de creencias, costumbres y modos de hacer las cosas de cada comunidad. La transmisión del legado es llamado por Herder tradición y es entendido como "un proceso continuo, el cual, por su propia naturaleza [...] envuelve una continua amalgama entre lo viejo y lo nuevo"78, un proceso dialéctico de apropiación y transformación. El lenguaje, entonces, es el medio a través del cual es posible la tradición y, en cuanto tal, lleva en su interior las características internas y cambiantes de cada pueblo ${ }^{79}$.

Ahora bien, estas reflexiones sobre la particularidad de cada pueblo son también el origen del concepto de conciencia popular. Herder explica que si bien la disposición natural de los seres humanos no es la guerra, entre los pueblos existen ambiciones y envidias basadas en su propio orgullo y honor. Este hecho hace que los pueblos sean susceptibles de enfrentarse entre $s^{i 80}$. Para Herder el conflicto cumple una función vital al estrechar los lazos internos de cada nación ${ }^{81}$. Solo ante la posibilidad del conflicto cada individuo siente de manera más fuerte que el pueblo es ese ente orgánico guiado por la tradición y que él mismo solo es una parte de su grandeza ${ }^{82}$. Esto supone que el conflicto hace más necesario que el pueblo mantenga el sentimiento de una identidad colectiva por medio de la preservación del carácter distintivo de su lenguaje. En ese sentido, lo que mantiene la unidad de los pueblos es la existencia de un espíritu nacional o conciencia popular representado por la creencia de que cada miembro del pueblo comparte un legado cultural común ${ }^{83}$. En este

\footnotetext{
74 BARNARD (2010) p. 22.

75 Herder (2010a) p. 165. "Since the whole human race is not one single homogeneous group, it does not speak one and the same language. The formation of diverse national languages, therefore, is a natural corollary of human diversity".

76 Herder (2010b) p. 299.

77 BaRnARD (2010) p. 31. "[...] relational event, a historical and cultural continuum” (énfasis en el original).

78 BARNARD (2010) p. 23. "[...] an ongoing process which, by its very nature [...] entails the continuous merging of the old and the new" (énfasis en el original).

79 Herder (2010a) p. 167.

80 Herder (2010a) pp. 167-168.

81 Herder (2010a) p. 176. Herder (2010b) p. 319.

82 Herder (2010a) p. 168.

83 Barnard (2010) pp. 29-30.
} 
legado cultural común el compartir un lenguaje cargado con la herencia de cada pueblo es vital.

La cuarta ley de la naturaleza expuesta por Herder establece que "dado que la humanidad forma con toda seguridad una totalidad en progreso, el cual se origina en un lugar común dentro del orden universal, todas las lenguas, y, con ellas, la cadena cultural, se derivan de una fuente común" ${ }^{84}$. Herder argumenta que dado que la humanidad tiende a un progreso continuo, cada uno de los elementos que forma la conciencia del pueblo también debe progresar. El progreso de cada pueble dependerá de qué tan arraigadas están sus $\operatorname{costumbres}^{85}$, por lo que es fácil encontrar pueblos más desarrollados que otros. Herder, entonces, plantea una relación directa entre la fortaleza de la conciencia popular y el desarrollo de cada pueblo.

La idea de conciencia popular o espíritu nacional que surge de esta teoría sobre el origen del lenguaje adquiere una connotación interesante en la forma en la que Herder piensa la organización política. Herder parte de la premisa de que una comunidad determinada solo puede ser considerada como una nación o un pueblo si tiene una cultura nacional propia y un lenguaje propio ${ }^{86}$. De ese modo, dado que el lenguaje es el medio a través del cual es posible la tradición, y es, a su vez, una característica determinante para la existencia del pueblo en cuando tal, las concepciones de cada pueblo viven y se reafirman a través del lenguaje. Estas concepciones son heredadas y no creadas por cada pueblo. Las costumbres, que para Herder hacen parte de estas concepciones propias de cada pueblo, hacen que el ser humano sea consciente de que depende de los demás y de la fuerza vivificante del pueblo para desarrollar sus capacidades ${ }^{87}$. De ese modo, la organización política, que para el autor es la forma en la que se organizan las capacidades de cada individuo en función de la comunidad, solo es posible por el establecimiento de unas reglas sustentadas en la tradición y en la cultura compartida de cada nación ${ }^{88}$. Esta conclusión es interesante porque lleva a Herder a pensar que los territorios en los que no existe una cultura compartida o en los que las reglas se han impuesto arbitrariamente no tienen una organización política sólida y están sujetos a la destrucción ${ }^{89}$.

La construcción de este argumento de Herder es interesante porque muestra una amalgama entre un enfoque normativo y otro descriptivo. La teoría sobre el origen del lenguaje y la idea de conciencia popular que se deriva de ella están basadas en la observación y la fisiología del ser humano. Sin embargo, al hablar de la organización política, Herder mezcla estos resultados descriptivos con recomendaciones sobre la organización correcta y fuerte en las cuales se tienen en cuenta las características definitorias de cada pueblo. Herder, entonces, no argumenta, al menos principalmente, que las normas que forman la

\footnotetext{
84 Herder (2010a) p. 170. "Since mankind in all probability forms one progressive totality, originating from one common origin within one universal order, all languages and, with them, the whole chain of culture, derive from one common source".

85 Herder (2010a) p. 173.

86 Herder (2010b) p. 284.

87 Herder (2010b) p. 311.

88 Herder (2010b) p. 317.

89 Herder (2010b) p. 324
} 
organización política de cada pueblo nazcan naturalmente de sus tradiciones y tengan su base en las costumbres, sino que afirma que eso debe ocurrir así si la organización política quiere ser sólida. En ese sentido, si bien pueden existir casos exitosos en lo que las normas nacen espontáneamente de la conciencia de cada pueblo, existen otros en los que ese proceso no ha ocurrido de esa manera. Tal vez, Herder llegó a su conclusión sobre las normas y la organización política al observar casos exitosos y compararlos con casos fallidos a la luz de su teoría sobre el carácter específico de cada pueblo y la conciencia popular o espíritu nacional. Ahora bien, la forma en la que ocurriría el proceso de adecuación o de creación normativa no es descrita por Herder.

Con todo lo anterior en mente se pueden buscar las coincidencias entre Savigny y Herder. Del mismo modo, este ejercicio de comparar estas dos formas de pensar puede ayudar a comprender mejor el pensamiento de Savigny.

\subsection{SAVIGNy y Herder}

El primer aspecto que se debe resaltar es la desconfianza en la noción de razón. Así, mientras Savigny desconfía de la noción de razón para hablar del origen del derecho, Herder desconfía de ella al teorizar sobre el desarrollo del lenguaje. En ese sentido, como lo afirma Knudson, Savigny critica la idea de que el derecho positivo pueda deducirse de unos principios eternos del derecho natural. En ese sentido, el derecho natural nunca podría ser el fundamento de validez del derecho positivo ${ }^{90}$. Por su parte, Herder procede de una manera distinta. Dado que el autor sitúa el origen del lenguaje en el hecho de que los seres humanos tienen conciencia de los objetos que perciben sus sentidos, en cierta medida está apelando a una noción de razón como introspección. En otros términos, la noción de razón es necesaria para su argumento, a pesar de que acuña el término mente reflexiva por considerar que la idea de razón no es correcta para referirse a las fuerzas del entendimiento humano ${ }^{91}$. Ahora bien, en relación con el desarrollo del lenguaje Herder resalta su relación con la conciencia popular y la actuación de fuerzas invisibles, espontáneas e irracionales.

El segundo aspecto que se debe resaltar son las nociones de pueblo y conciencia popular de los dos autores. Es claro que Savigny y Herder piensan al pueblo y a la conciencia popular de la misma manera. Así, ambos conciben al pueblo como un todo orgánico con una identidad propia que es observable a través de signos externos como las costumbres. Del mismo modo, ambos piensan, implícitamente en el caso de Savigny, que las costumbres son transmitidas por una tradición que solo es posible por la existencia del lenguaje. De hecho, Savigny se apropia explícitamente de la tesis de Herder sobre la forma en la que el pueblo reafirma su unidad a través del lenguaje al afirmar que "[el pueblo es] una comunidad espiritual que en el uso del mismo lenguaje se evidencia, robustece y desarrolla"92. Del mismo modo, al leer a los dos autores de forma conjunta se puede pensar que la forma en la que Herder desarrolla la noción de pueblo y la forma en la que nace la categoría de

\footnotetext{
90 Knudson (2002) p. 411

91 Herder (2010a) pp. 131-132. Barnard (2010) p. 20.

92 SAVIGNY (1949) p. 39.
} 
conciencia popular sirven para explicar el contenido de esos conceptos en la teoría de Savigny.

Ahora bien, esta correspondencia en la forma de pensar el pueblo y la conciencia popular le agrega elementos a la teoría de Savigny que no son evidentes en una lectura de este autor. Savigny es claro al afirmar que existe un carácter equivalente o analógico entre el derecho y el lenguaje de cada pueblo ${ }^{93}$. Esta forma de pensar de Savigny es interesante porque le da al derecho una dimensión existencial; es decir, lo relaciona con la existencia misma del pueblo en cuanto tal. Así, mientras Herder afirma que una comunidad solo puede considerarse como una nación o un pueblo en la medida en que tenga una cultura nacional propia y un lenguaje propio ${ }^{94}$, Savigny diría que esa misma comunidad solo es un pueblo si tiene un derecho propio. En ese sentido, la crítica de Savigny a una codificación que no se guíe por un sentido histórico y científico ${ }^{95}$ adquiere un nuevo matiz: si la codificación en tanto compendio de todo el derecho y unificación de fuentes ${ }^{96}$ no se realiza correctamente, podría poner en peligro la existencia misma del pueblo. Por otra parte este rasgo de Savigny puede agregar elementos a la crítica que realiza Franklin al compromiso conservador y feudal que encuentra en este autor ${ }^{97}$.

De forma complementaria a lo anterior, Herder puede ayudar a entender la afirmación de Savigny de que el derecho reside en la conciencia popular de una manera distinta. Kantorowicz al comentar esta idea afirmó que es un aspecto que convierte a la teoría de Savigny principalmente en una descripción sociológica ${ }^{98}$. En el mismo sentido Reimann ${ }^{99}$ y Kutner ${ }^{100}$ resaltan el potencial descriptivo de esta idea en la medida en que es razonable pensar que el derecho refleja las necesidades concretas y el carácter particular de cada pueblo. Sin embargo, de la mano de Herder se podría decir que esta idea tiene también una dimensión normativa. Solo de ese modo el carácter existencial del derecho en tanto análogo al lenguaje adquiriría un sentido pleno. Si bien Savigny es claro al afirmar que el derecho nace espontáneamente de la conciencia popular, es decir, que se crea por las costumbres y las creencias populares ${ }^{101}$, utiliza este mismo argumento para juzgar la calidad de la ley en tanto expresión visible del derecho ${ }^{102}$. Así, del mismo modo en que Herder afirma que la organización política exitosa es aquella basada en normas sustentadas en la tradición ${ }^{103}$, Savigny diría que dado el especial carácter del derecho, las buenas leyes son aquellas que expresan plenamente la conciencia del pueblo y favorecen su conservación.

\footnotetext{
93 SaVigny (1949) pp. 34, 36. SAVIGNY (1977) pp. 44, 46.

94 Herder (2010b) p. 284.

95 SaVigny (1977) pp. 56, 142.

96 SaVigny (1977) p. 52.

97 FrankLin (1952) pp. 68-71.

98 Kantorowicz (1937) p. 334.

99 Reimann (1990) p. 852.

100 Kutner (1972) p. 284.

101 SAVIGNY (1977) p. 48.

102 SAVigny (1949) p. 31.

103 Herder (2010b) p. 317.
} 
La reflexión anterior se complementa si se tiene en cuenta la siguiente afirmación de Savigny: "Según la teoría de los otros [los miembros de la Escuela Histórica], no se da ninguna existencia humana completamente individual y separada; antes bien, aquello que puede ser considerado como individual, ha de mirarse, por otra parte, como miembro de un todo superior" ${ }^{104}$. En este pasaje Savigny cita explícitamente la reflexión de Herder sobre la relación entre el individuo y su pueblo ${ }^{105}$. En ese sentido, Savigny no solo tendría un compromiso normativo con la conservación del pueblo, sino también con la conservación del individuo en tanto miembro de una unidad superior.

La implicación de las dos ideas presentadas anteriormente puede ser discutible para los estudiosos que se esfuerzan en ver una separación entre el método de Savigny y el método del derecho natural ${ }^{106}$. Anteriormente se dijo que el método del derecho natural mezclaba de forma incorrecta elementos jurídicos con principios morales y políticos. Ahora bien, observar que la teoría de Savigny sobre el origen del derecho tiene una dimensión normativa hace pensar en un compromiso político de esta idea. Entonces, la conservación del pueblo es un principio político que está al interior de la teoría de Savigny y hace que su forma de pensar la ciencia jurídica no esté privada del todo de filosofía jurídica. En ese sentido, Savigny no logra apartarse del todo del método del derecho natural en la forma en la que lo expone Reimann ${ }^{107}$ por la existencia de un compromiso normativo en la construcción de la ciencia del derecho ${ }^{108}$. Tener en cuenta esta circunstancia es el primer paso para observar la tensión entre particularismo y universalismo en Savigny.

\section{LA SEPARACIÓN ENTRE SAVIGNY Y HERDER Y LA TENSIÓN ENTRE PARTICULARISMO Y UNIVERSALISMO}

La categoría de ciencia jurídica no ha estado presente en este escrito hasta este momento. Esto se ha hecho de forma intencional para resaltar que la forma en la que Savigny llega a esta noción es el principal punto de separación entre su teoría y la de Herder. Atrás se enfatizó que Savigny plantea una diferencia entre el origen y el desarrollo del derecho. En ese sentido, Savigny explica que en los estados avanzados de la civilización el derecho se convierte "en materia de la competencia de los juristas, que en tal concepto vienen a representar al pueblo"109. De ese modo, el desarrollo del derecho debe pasar necesariamente por la elaboración científica de los juristas, quienes se convierten en los catalizadores de la conciencia popular ${ }^{110}$. La aparición del jurista y con él la ciencia del derecho plantea para Wintgens la existencia de una paradoja: mientras Savigny critica el método racionalista

104 Dufour (1981) p. 23. La cita corresponde al ensayo introductorio de Savigny a la Revista para la Ciencia Histórica del Derecho.

105 Herder (2010a) p. 168.

106 REIMANN (1990).

107 ReImANn (1990) pp. 842-846.

108 Cfr. Gómez (1999). El autor hace un recuento de los argumentos que se han dado a favor y en contra del "iusnaturalismo" de Savigny.

109 SaVigny (1949) pp. 37-38. SaVigny (1977) p. 47.

110 SAVIGNY (1949) p. 60. 
abstracto basado en el derecho natural que fue usado en la codificación francesa y enfatiza el origen irracional del derecho ${ }^{111}$, ahora enfatiza la necesidad del tratamiento científico del derecho como una muestra del proceso civilizador ${ }^{112}$.

Esto muestra que para Savigny, mientras el origen del derecho se encuentra en la conciencia popular, su estudio y, más aún, su desarrollo son principalmente científicos y racionales. Esta circunstancia indica que la relación entre el derecho y el lenguaje y, consecuentemente, entre las teorías de Savigny y Herder no es tan perfecta o pacífica como lo pretenden mostrar los autores citados más arriba. Esta relación tiene un límite: la intermediación del jurista para observar la conciencia popular y representar al pueblo. Savigny llega a aceptar la posibilidad de que el trabajo científico del jurista sea la única fuente de derecho en una sociedad determinada. Esto sucedería en la medida en que la producción espontánea de derecho por la conciencia popular es cada vez más difícil cuando la humanidad sale de su estado primitivo ${ }^{113}$. Incluso, si en una sociedad prima el elemento científico por encima del político, este último podría ser opacado completamente. Esta intermediación del jurista por medio de la elaboración científica del derecho riñe con la postura antirracionalista de Herder y hace más evidente la paradoja que plantea Wintgens. Es más, si bien Herder acepta un proceso de adecuación de las normas a las costumbres para poder construir una organización política sólida, cuesta mucho trabajo conciliar la idea de un intermediario de la conciencia popular con sus tesis sobre la reafirmación espontánea de los lazos populares ante la inminencia de un conflicto o la tradición. En ese sentido, si bien Savigny y Herder tienen posturas antirracionalistas ${ }^{114}$, Herder no aceptaría que el desarrollo de un rasgo característico de la conciencia popular esté sujeto a una elaboración racional por parte de un grupo privilegiado de personas. Ahora, es claro que si bien la descripción que realiza Herder es un ejercicio racional, el fenómeno que describe es esencialmente irracional.

La introducción de la ciencia del derecho es, como se dijo atrás, un paso analítico desconcertante de Savigny.

En De la vocación de nuestro siglo Savigny desarrolla la noción de ciencia del derecho de forma muy cercana a su teoría sobre el origen del derecho positivo. Allí, Savigny afirma que un buen código solo puede ser construido científicamente, lo que significa que los juristas deberían identificar los principios jurídicos presentes en la comunidad y la forma en la que ellos se conectan entre si $^{115}$. En este caso, si bien es evidente la intermediación, se conserva la tesis inicial, es decir, que el derecho es esencialmente costumbre y emana del pueblo $^{116}$. No se puede dejar de lado que si bien se conserva la tesis inicial, la forma en la que se desarrolla es muy distinta a como lo haría Herder. Lo anterior se repite en el Sistema de derecho romano actual cuando Savigny resalta la dimensión material del trabajo del

\footnotetext{
111 SAVIGNY (1977) p. 41.

112 Wintgens (2010) pp. 95-96.

113 SAVIGNY (1949) p. 38.

114 Cfr. Dufour (1981) p. 17. El autor ubica la teoría de Savigny dentro de la "vasta corriente de restauración de los valores irracionales que irrumpe hacia fines del siglo XVIII”.

115 Savigny (1977) p. 56.

116 Reimann (1990) p. 852
} 
jurista por "concentrarse en ellos principalmente la producción jurídica del pueblo, la que practican continuamente como representantes de la totalidad" 117.

Estos argumentos se observan bien en la relación entre la ciencia del derecho y la legislación. En ese contexto Savigny afirma que "el derecho positivo encarnado en el lenguaje y provisto de poder absoluto se denomina ley y en su establecimiento pertenece a los derechos más nobles del poder supremo del Estado" ${ }^{118}$. Con respecto a la definición de ley que se había citado en este escrito (la ley como expresión visible del derecho), en el contexto de la relación entre la legislación y la ciencia del derecho Savigny introduce elementos adicionales: el Estado y el poder absoluto de la ley. La razón de estos nuevos elementos es explicada por Savigny en Metodología jurídica. El autor explica que el Estado, que, como se dijo antes, es la aparición visible y orgánica del pueblo concentrada en fronteras determinadas ${ }^{119}$, obedece a la necesidad de limitar la arbitrariedad entre los individuos. Dado que esta función no podría ser puesta en manos de un tercero porque entonces el pueblo se sometería a su arbitrariedad, "era mejor que existiese algo del todo independiente y alejado de toda convicción individual: la ley" ${ }^{\prime 20}$. La ley, sin embargo, al ser una característica del derecho en el Estado y, por tanto, en el estado avanzado de la civilización, es siempre establecida por la ciencia del derecho ${ }^{121}$. En ese sentido, las funciones de la ley deben ser coherentes con la conciencia popular que es, en última instancia, el origen del derecho positivo. La ciencia del derecho, entonces, al crear la ley debe hacer de ella un instrumento complementario al derecho positivo y de apoyo a su progreso ${ }^{122}$. Así, la legislación debe aclarar los puntos indeterminados de la costumbre e introducir en el derecho antiguo los cambios que se observen en las costumbres, opiniones o necesidades del pueblo ${ }^{123}$. Este rasgo de la teoría de Savigny hace pensar a Mollnau que el autor efectivamente tiene una teoría de la legislación que merece ser tenida en cuenta por aquellos que se concentran en la teoría sobre el origen del derecho positivo. La influencia de la ciencia del derecho en la legislación, como lo muestra Mollnau, es evidente en tanto el jurista debe identificar los rasgos del derecho propio para introducir cambios de forma orgánica en su interior y no perturbaciones del derecho en su totalidad ${ }^{124}$. No sobra decir que en esta formulación la preservación de la existencia del pueblo de Herder y la organización política adquieren un aspecto interesante: es necesario tener un orden externo e independiente de los individuos representado en la ley para evitar los excesos de poder entre ellos y la destrucción interna del pueblo.

Lo que se ha dicho hasta ahora sirve para observar que en esta primera formulación de la ciencia del derecho en la que se enfatiza su dimensión material Savigny expresa con toda su magnitud el componente normativo de su tesis sobre el origen del derecho positivo. Del mismo modo, el autor expresa su compromiso político con la conservación del pueblo.

117 SAVIGNY (1949) p. 60.

118 SAVIGNY (1949) p. 54.

119 SAVIGNY (1949) p. 41.

120 SAVIGNY (2004) p. 19.

121 SAVIGNY (2004) p. 19.

122 SAVIGNY (1949) p. 55.

123 Savigny (1949) pp. 55-56. SaVigny (1977) pp. 51-52.

124 Mollnau (1989) p. 88-89. 
En toda esta formulación Savigny se ha preocupado porque los resultados de la ciencia del derecho, en tanto catalizadora del desarrollo del derecho, sean siempre coherentes con las particularidades del pueblo en el que se inserta. En otras palabras, esta aproximación a la ciencia del derecho permitiría pensar a Savigny como un teórico que le presta atención a las particularidades de cada pueblo y la forma en la que deben retratarse en el derecho. Este particularismo de Savigny está relacionado también con el principio extrajurídico de la preservación de la existencia del pueblo. No está de más recordar que esta dimensión del pensamiento del autor es la que más se reseña en los artículos de divulgación de su teoría.

El particularismo de Savigny tiene tres características principales. En primer lugar, Savigny tiene en su cabeza la idea de que el derecho es esencialmente un fenómeno cultural que está sometido al cambio a través del tiempo. En ese sentido, el derecho presente es el producto de la historia ${ }^{125}$. En tanto fenómeno cultural, como ya se dijo, el derecho tiene una relación interna y necesaria con cada pueblo en particular. En segundo lugar, Savigny establece que el derecho no solo está sometido a un cambio constante sino a una evolución ${ }^{126}$. La evolución, como se vio con Herder, es la característica de una nación con una conciencia sólida. Savigny se adhiere a esta tesis cuando afirma que los pueblos cambian a través de la historia hasta llegar a estados cada vez más avanzados en términos culturales e intelectuales ${ }^{127}$. Ahora, dado que el desarrollo del derecho propiciado por el jurista se basa en los cambios internos de la conciencia del pueblo, este desarrollo debe reflejar la evolución de cada pueblo. La profunda conexión entre el desarrollo del derecho y la evolución de un pueblo supone que el derecho se piensa en términos orgánicos ${ }^{128}$. A su vez, una conclusión como la anterior que depende de una concepción orgánica de la historia ${ }^{129}$. En tercer lugar, pensar el derecho como un fenómeno cultural propio de cada pueblo que está sujeto a una evolución junto con él implica prestarle atención a los hechos jurídicos y no a construcciones racionales abstractas ${ }^{130}$. En ese sentido, el jurista debe tener en cuenta que el derecho va más allá de la arbitrariedad de cada individuo. El componente normativo de cada una de estas características es evidente al observar que Savigny las pensaría como necesarias para la construcción de un ordenamiento jurídico que propicie la conservación del pueblo.

A pesar de lo anterior, Savigny identifica una dimensión adicional de la ciencia del derecho. El autor afirma que se trata de "una actividad formal, puramente científica, por llevar a la conciencia y exponer científicamente el derecho, no importa el origen que haya tenido" ${ }^{131}$. El aspecto formal del derecho no tiene que ver con la elaboración de la legislación y el desarrollo del derecho sino que tiene que ver con el trabajo directo con la ley y su aplicación práctica ${ }^{132}$.

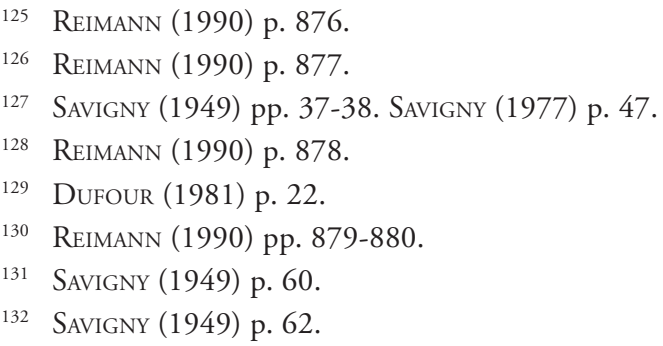


La diferencia entre la dimensión material y la dimensión formal de la ciencia del derecho se entiende mejor por intermedio de la teoría de la interpretación de Savigny, la cual es una de las herramientas de la dimensión formal. El objeto de la interpretación ya no es el derecho sino la ley, en los términos en que se definió al relacionar la legislación con la ciencia del derecho ${ }^{133}$. De forma novedosa Savigny afirma que el objeto principal de la interpretación no es llenar de contenido aspectos oscuros o confusos de la ley sino reconstruir el pensamiento expresado en la ella ${ }^{134}$. La finalidad de la interpretación es facilitar la aplicación práctica de la ley. De ese modo "la actividad libre contenida en la interpretación tiende a que conozcamos la ley en su verdad, es decir, que se nos hace evidente mediante un procedimiento regular. La interpretación de la ley es siempre necesaria para que intervenga en la vida; y esta su general necesidad constituye a la par su justificación"135.

La labor de la interpretación tiene dos partes: una elaboración sistemática y otra elaboración histórica. En ese sentido, para poder mostrar el contenido de la ley será necesario buscar los principios de los cuales se deriva como resultado de un desenvolvimiento histórico; del mismo modo, es necesario ubicar la ley de forma coherente en una unidad ${ }^{136}$. Sin embargo, Savigny enfatiza con mayor medida el elemento sistemático de la interpretación al dedicar un gran espacio de su teoría a pensar la analogía, las lagunas y la forma de corregir leyes defectuosas ${ }^{137}$. Además, el autor justifica este tratamiento por la necesidad de tener un ordenamiento jurídico perfecto y unificado que "sea capaz de resolver cualquier problema existente en el campo del derecho"138. Esta particularidad de la interpretación le sugiere a Reimann que Savigny piensa que la búsqueda de los principios fundamentales de la ley por medio del análisis histórico, es decir, por medio de la dimensión material de la ciencia del derecho, es un paso previo para la verdadera teoría de la interpretación y la ciencia del derecho: la construcción de un sistema jurídico científico y coherente ${ }^{139}$. Si bien la opinión de Reimann no le hace justicia a la magnitud de la dimensión material de la ciencia del derecho de la que se habló antes, sí se puede decir a partir de él que la principal tarea del enfoque formal de la ciencia del derecho es construir un sistema jurídico con las leyes existentes y no necesariamente promover el desarrollo del derecho positivo.

De lo anterior se puede decir que la oposición entre la dimensión material y la dimensión formal de la ciencia del derecho es la contraposición entre organicismo y sistematicismo. Mientras la dimensión material hace de la ciencia del derecho un medio para expresar el aspecto vital, actuante y evolutivo del derecho en tanto expresión de la conciencia popular, la dimensión formal deja de lado esta idea para brindar una herramienta que faci-

\footnotetext{
133 SAVIGNY (2004) p. 17.

134 SAVIGNy (2004) p. 20. Cfr. BARría (2011). En este artículo el autor hace una exposición distinta de la teoría de la interpretación de Savigny. Por su propósito, el autor decide enfatizar los elementos de interpretación de Savigny, a saber: el elemento gramatical, el elemento lógico, el elemento histórico y el elemento sistemático.

135 SAVIGNY (1949) p. 78.

136 SAVIGNY (2004) pp. 21, 25. Zuleta (1977) p. 71

137 SAVIGNY (1949) pp. 90-152.

138 SAVIGNY (1949) p. 123.

139 Reimann (1990) p. 354.
} 
lite el uso práctico y operativo del derecho al proveer un sistema del cual se puedan obtener las respuestas a todos los casos posibles.

Esta dimensión sistemática del derecho tiene tres implicaciones importantes. En primer lugar, pensar así supone que el sistema es el orden inherente en el derecho positivo y la ley ${ }^{140}$. Savigny es claro al afirmar que la exposición que realice la ciencia del derecho en su dimensión formal debe mostrar que el derecho positivo y la ley forman una unidad caracterizada por el orden y la coherencia. De ese modo, la labor del jurista al construir el sistema es reflejar el orden interno que es inherente en las fuentes del derecho ${ }^{141}$. Wintgens explica que pensar el mundo en términos sistemáticos es un remanente de la filosofía cartesiana, de la cual Savigny hace una referencia explícita. Descartes desarrolla esta idea con dos argumentos principales. Por un lado, el ser humano solo puede ser consciente con certeza de su propia existencia en tanto ser pensante. Por otro, la anterior idea solo puede ser verdadera si Dios garantiza la conexión entre certeza y verdad. En ese sentido, el conocimiento de las cosas será verdadero y podrá representar el mundo tal y como es si las cosas mismas tienen, de hecho, una existencia ordenada. Esta existencia ordenada solo puede ser garantizada por Dios como primer principio ${ }^{142}$. En segundo lugar y como consecuencia de la finalidad operativa de la dimensión formal de la ciencia jurídica, el sistema es perfecto y completo ${ }^{143}$. Esta perfección y completitud supone la existencia de axiomas a partir de los cuales el jurista práctico pueda deducir las respuestas a los casos a los que se enfrente. En tercer lugar, y como consecuencia de la oposición entre organicismo y sistematicismo, el sistema que construye el jurista parece ser estático ${ }^{144}$. Esta estabilidad se muestra por el deber que tiene el sistema de tener axiomas necesarios para la aplicación práctica del ordenamiento jurídico. Estos axiomas, al formar la base de un sistema completo y lógico, no pueden estar sujetos a la contingencia.

Ahora bien, lo interesante de observar el carácter y las implicaciones de la dimensión formal de la ciencia del derecho de Savigny es darse cuenta de que después de exponer su teoría de la interpretación en el Sistema de derecho romano actual, el autor se dedica exclusivamente a construir el sistema que el título del libro promete. Sin embargo, antes de hablar propiamente del derecho romano, Savigny se refiere a la naturaleza de las relaciones jurídicas en general. Es decir, para construir su sistema, Savigny empieza definiendo los axiomas que le permitirán darle orden al derecho privado romano. Cualquier lector que haya pasado por las primeras páginas del Sistema o De la vocación de nuestro siglo esperaría que Savigny empezara su argumentación apelando a los principios históricos y, por lo tanto, a la conciencia del pueblo para poder explicar la naturaleza de las relaciones jurídicas. Es más, este mismo lector esperaría que Savigny empezara a hablar de las relaciones jurídicas en el derecho romano germánico, lo que sería lógico si se tiene en cuenta que Savigny

140 Reimann (1990) p. 880. Wintgens (2010) p. 96.

141 Wintgens (2010) p. 86

142 Wintgens (2010) pp. 87-88.

143 Reimann (1990) p. 881.

144 Reimann tiene una opinión distinta. De acuerdo con él, la forma en la que piensa Savigny el sistema es flexible y orgánica. Cfr. Reimann (1990) p. 882. Más adelante en este escrito se verá que los axiomas que Savigny construye como fundamentos de su sistema permiten contradecir esta conclusión. 
afirma que fue el espíritu alemán el que moldeó el derecho romano de su época ${ }^{145}$. En lugar de esto, el lector se encuentra con la siguiente premisa: "El hombre se encuentra rodeado del mundo exterior; y el elemento más importante de este [sic] su mundo circundante es el contacto con aquellos [sic] que le son iguales por su naturaleza y destino. Ahora bien; para que sea posible que seres libres coexistan fomentándose mutuamente en su desenvolvimiento sin estropearse, es menester el reconocimiento de una frontera invisible dentro de la cual la existencia y la eficacia de cada individuo logre un campo de acción seguro y libre. La regla por la cual se determina aquella frontera y a través de ella este espacio libre, es el derecho [...]. Desde el punto de vista ahora asegurado, concebimos cada relación jurídica individual como una relación entre una persona y otra persona determinada por una regla jurídica. La determinación por una regla jurídica consiste en que se indica a la voluntad individual un campo en el cual ha de reinar con independencia de toda voluntad ajena" ${ }^{146}$.

Este pasaje es realmente desconcertante, más aún si se tiene en cuenta que esta es la idea que guiará gran parte de la exposición de Savigny sobre la sistematización de las relaciones jurídicas en el derecho privado. Este punto de partida hace pensar a Reimann que la concepción formal del derecho de Savigny es realmente individualista dado que se considera al derecho en relación con la libertad individual y no con las relaciones al interior de cada nación ${ }^{147}$ o el desarrollo orgánico del pueblo. Se puede ir más allá y afirmar con Kennedy que esta premisa, que es el axioma que permite construir el sistema jurídico, es esencialmente universalista ${ }^{148}$. Más aún, este universalismo se puede explicar por la necesidad de buscar una proposición que sirva como base al sistema y que no esté sujeta a la contingencia ${ }^{149}$.

El universalismo de Savigny al construir el sistema es más evidente cuando desarrolla qué quiere decir que el derecho determina un campo en el que la voluntad individual puede reinar. Savigny explica que la voluntad individual puede dirigirse hacia la propia persona o hacia el mundo exterior ${ }^{150}$. Mientras que en el primer caso el ser humano tiene un poder legítimo sobre sí mismo y sus fuerzas, en el segundo caso las cosas y las personas ajenas son los objetos de la voluntad ${ }^{151}$. La unidad de análisis de Savigny para construir el sistema deja de ser el pueblo; ahora es el sujeto que expresa su voluntad el que toma su lugar. La univer-

\footnotetext{
145 SaVigny (1977) pp. 70-71. Kantorowicz (1937) p. 338.

146 SaVigny (1949) pp. 181-183.

147 Reimann (1990) p. 852.

148 Kennedy (2010) p. 812.

149 Cfr. Blasco (1999) pp. 12-13. La autora realiza una lectura interesante de este rasgo de la teoría de Savigny: "La elaboración de una parte general del derecho civil significa sistematización, abstracción e intemporalidad de los conceptos esenciales del derecho [...] Esta 'historicidad' del derecho de Savigny permite su abstracción e intemporalidad y, por tanto, la creación de una parte general en la que los agentes del derecho sean el sujeto de derecho; los distintos patrimonios, el patrimonio; y las distintas relaciones jurídicas, la relación jurídica. Su justificación está en la historia” (énfasis en el original). Sin embargo, en la exposición de Blasco, no es clara la conexión entre la historia y la sistematización.

150 Sobre la importancia de la noción de voluntad en la teoría del perfeccionamiento del contrato en Savigny, véase Neme (2012). Si se lee este artículo teniendo en cuenta lo que se ha dicho hasta ahora en este escrito, es posible notar la importancia de una noción universalista de voluntad en la dogmática del derecho civil de Savigny.

151 SAVIGNY (1949) pp. 184-187.
} 
salidad del sujeto y del sistema que sustenta es su yo, que tiene la voluntad como contenido más allá de cualquier determinación histórica. Esta es una de las razones por las que Kennedy ubica a Savigny dentro de lo que denomina tradición jurídica clásica ${ }^{152}$. De ese modo, parecería que la tesis sobre el origen y la evolución del derecho que Savigny desarrolla en los primeros parágrafos del Sistema y De la vocación de nuestro siglo se diluye o se deja en suspenso ${ }^{153}$.

Si se tiene en cuenta que Savigny llega a la distinción entre derecho patrimonial y derecho de familia a partir de los entes hacia los cuales el sujeto dirige su voluntad ${ }^{154}$ y que esta distinción es vital para la organización de su sistema ${ }^{155}$, es claro que una tensión entre particularismo y universalismo atraviesa la teoría de Savigny. Esta tensión existe porque tanto la dimensión material como la dimensión formal de la ciencia del derecho son formas válidas de practicarla; más aún, si se recuerda que tanto el orden que generan los resultados del enfoque formal como el progreso del derecho que facilita y permite el enfoque material son necesarios. Con esa tensión en mente se puede entender mejor la tensión entre reglas absolutas y reglas mediadoras que introduce Savigny y de la cual no se ha hablado hasta ahora. Savigny escribe: "Analicemos la relación en la que se encuentran las reglas jurídicas con las relaciones jurídicas por ellas denominadas [...]. En este punto existe la siguiente oposición. Una parte de las reglas jurídicas debe reinar con necesidad inalterable, sin dejar campo a la acción individual. Las denomino reglas jurídicas absolutas o imperantes. El fundamento de esa necesidad puede estribar en la naturaleza del mismo ordenamiento jurídico conforme se expresa en el derecho positivo, o en fines políticos y de economía política o también inmediatamente en consideraciones morales [...]. Otra parte de las reglas concede en principio libre poder a la voluntad individual; y solo donde la voluntad ha dejado de ejercer su poder se coloca la regla jurídica en su lugar para dar a la relación jurídica la necesaria determinación. Estas reglas a las cuales se puede considerar como interpretaciones de la voluntad que quedó imperfecta las denomino reglas mediadoras"156.

Las reglas absolutas, entonces, son el dominio de la dimensión material de la ciencia jurídica. En ese sentido la necesidad de estas normas guarda una íntima relación con el enfoque normativo resaltado más arriba. Así, las reglas absolutas, en tanto están relacionadas con las íntimas necesidades y creencias del pueblo, permiten su permanencia y desenvolvimiento. Por otra parte, las reglas mediadoras son el dominio del enfoque formal de la ciencia del derecho en tanto la idea de sistema está pensada para dar seguridad a las relaciones jurídicas.

La potencia de esta tensión es tan grande que incluso puede verse al revisar con más profundidad la forma en la que Savigny construye el sistema jurídico. Como se dijo, Savigny divide el derecho privado en derecho patrimonial, cuyas instituciones principales son la

152 Kennedy (2010) pp. 831, 833, 835.

153 Hoeflich (1989) pp. 23-24. El autor presenta algunas críticas que se formularon a la forma en la que Savigny empieza a construir la ciencia del derecho. Una de esas críticas indica que los escritos de Savigny son excesivamente teóricos y que tienen un escaso valor práctico para los "abogados modernos".

154 SaVigny (1949) pp. 188-190.

155 Kennedy (2010) p. 819.

156 SAVIGNy (1949) p. 69. 
propiedad y las obligaciones, y derecho de familia, cuyas instituciones fundamentales son el matrimonio, la patria potestad y la filiación. Kennedy explica esta distinción por medio de dos pares de principios que para Savigny reflejan las dimensiones del ser humano. El primer par de principios lo conforman la completitud y la incompletitud de la persona ${ }^{157}$. En el derecho patrimonial se muestra el ser humano como una "totalidad en sí misma cerrada, de suerte que cada una se [enfrenta] con la otra como con un ser completamente homogéneo" 158 . Es evidente que el ser humano del derecho patrimonial es el sujeto universal. Por su parte en la familia "[el individuo] no aparece como [...] un ser existente por sí sino como un miembro de la totalidad orgánica de toda la humanidad [...]. En ellas no aparece el individuo como en las obligaciones, como un ser independiente, sino como un ser incompleto necesitado de complemento en un gran contexto natural [...]. La relación familiar [tiene como objeto] a la persona como totalidad en cuanto es un miembro en el contexto orgánico de la humanidad entera" ${ }^{\text {"159. }}$.

Para Kennedy esta forma de pensar la familia, lejos de ser una descripción, es una forma de camuflar un aspecto jurídico sustancial ${ }^{160}$. Más allá de esto se puede decir que aquí Savigny está hablando del aspecto universalista de la familia. La familia aparece como una "institución natural" que al tener relación con el "contexto orgánico de la humanidad" se define de forma externa a cualquier consideración histórica o visión de un pueblo en particular.

El segundo par de principios lo conforma la necesidad y la arbitrariedad ${ }^{161}$. Así, mientras el derecho patrimonial está atravesado por el dominio pleno de la voluntad en lo que se refiere a la propiedad y la capacidad de obligarse ${ }^{162}$, el derecho de familia tiene una triple dimensión: natural, jurídica y moral ${ }^{163}$. Así, el carácter natural de la familia hace que cada sociedad deba tener necesariamente un derecho de familia ${ }^{164}$, y su carácter moral hace que el derecho de familia esté determinado por la concepción moral de cada pueblo y, por esa vía, por su conciencia ${ }^{165}$. Aquí la dimensión particularista de la familia hace su aparición: si bien es una institución natural, sus normas tienen relación con el carácter de cada pueblo. Del mismo modo, lo anterior hace que las normas sobre la familia sean absolutas. De hecho, si se hace eco de lo que se ha dicho hasta ahora sobre el enfoque normativo de Savigny se puede decir que una buena legislación de familia será aquella que tenga este aspecto particular en mente. Asimismo, aquí la conservación de la familia es otra forma de conservar la unidad del pueblo.

Lo interesante de la distinción entre derecho patrimonial y derecho de familia es que, en última instancia, es la expresión de la tensión entre particularismo y universalismo

157 Kennedy (2010) p. 814.

158 SaVigny (1949) pp. 188-189.

159 SaVIGnY (1949) pp. 189, 191.

160 Kennedy (2010) p. 815.

161 Kennedy (2010) p. 815

162 Savigny (1949) pp. 211, 213.

163 SaVigny (1949) pp. 193-194.

164 Kennedy (2010) p. 819.

165 SAVIGNY (1949) p. 194 nota (a). 
de Savigny en un contexto que parece estar dominado por la idea de un sujeto universal. Si bien Savigny empieza a pensar el derecho de familia a partir de su noción de sujeto, es claro que al desarrollar la idea y resaltar la conexión entre la familia y la conciencia popular, aparece la necesidad de que el derecho de familia obedezca a las particularidades de cada pueblo. Por otro lado, en tanto el derecho patrimonial está basado enteramente en una idea de sujeto impersonal que se dirige hacia el mundo exterior por medio de sus actos de voluntad, expresa muy bien la idea de universalismo que está implícita en la noción de un sistema como descripción completa del ordenamiento jurídico ${ }^{166}$.

\section{CONCLUSIONES}

En este escrito se pretendió abordar la relación entre el pensamiento de Savigny y la filosofía romántica de Herder. Para hacerlo, se aprovecharon las referencias explícitas de Savigny al origen del lenguaje y su analogía con el origen del derecho para buscar los puntos de contacto entre los dos autores. Tras hacer este ejercicio se encontró que las tesis de Herder llevan a comprender mejor algunas tesis de Savigny y a pensar en este último la existencia de elementos normativos que habían sido dejados de lado por otros estudiosos. Sin embargo, este ejercicio también mostró que si bien existen profundas conexiones entre los dos autores, el punto crítico de separación se encuentra en la introducción de la ciencia del derecho y el paso analítico que hizo este resultado posible. A partir de esta diferencia con Herder fue posible pensar y exponer la tensión entre particularismo y universalismo que atraviesa las dos dimensiones de la ciencia del derecho de Savigny. En última instancia, esta tensión parece sostenerse en el compromiso político de Savigny con la conservación de la unidad del pueblo. Del mismo modo, siguiendo la explicación de Reimann sobre el método del derecho natural ${ }^{167}$, la existencia de este elemento extrajurídico en la construcción de la ciencia del derecho permitiría afirmar que Savigny no se separó del todo del método del derecho natural.

166 Cfr. Hanisch (1980) pp. 171, 173. Al desarrollar la influencia de Savigny en la noción de persona jurídica que usa Andrés Bello en su Proyecto de Código Civil, el autor se refiere de forma implícita a esta diferencia entre el derecho patrimonial y el derecho de familia: "El carácter esencial de la corporación es que su derecho no reposa sobre sus miembros tomados irregularmente, ni aun sobre todos sus miembros reunidos, sino sobre un conjunto ideal".

167 Reimann (1990) p. 842. 


\section{BIBLIOGRAFÍA CITADA}

Barnard, Frederick M. (2010): "Introduction", en Barnard, Frederick M. (ed.), J. G. Herder on social and political culture (Cambridge, Cambridge University Press) pp. 3-60.

Barría, Manuel (2011): "El elemento de interpretación gramatical. Su origen en Savigny, algunos autores modernos y la doctrina nacional", Ars Boni et Aequi, vol. $7 \mathrm{~N}^{\circ}$ 2: pp. 257-282.

Blasco, Yolanda (1999): "Notas sobre la recepción de la parte general de Savigny en España”, Cuadernos del Instituto Antonio de Nebrija, No 2: pp. 11-36.

Dafour, Alfred (1981): "Savigny y el pensamiento del Siglo XVIII", Revista Persona y Derecho, $\mathrm{N}^{\circ}$ 8: pp. 11-32.

Engel-Janosi, Friedrich (1947): “The Intellectual Background of Savigny”, Seminar (Jurist), vol. 5: pp. 39-61.

Fernández-Crehuet, Federico (2008): La perspectiva del sistema en la vida y obra de Friedrich Carl von Savigny (Granada, Editorial Comares).

Franklin, Mitchell (1952): "The Kantian Foundations of the Historical School of Law of Savigny", Revista Jurídica de la Universidad de Puerto Rico, vol. 22 No 1-4: pp. 64-89.

Franklin, Mitchell (1970): "Legal Method in the Philosophies of Hegel and Savigny", Tulane Law Review, vol. 44 N 4: pp. 766-797.

Gómez, Juan Antonio (1999): "El problema del Derecho Natural en la doctrina historicista del derecho de F. K. von Savigny”, Anuario de Filosofía del Derecho, N 16: pp. 253-276.

Hanisch, Hugo (1980): "La influencia de Savigny en Bello en materia de personas jurídicas”, Revista de Estudios Histórico-Jurídicos, N 5: pp. 167-198.

Herder, Johann Gottfried von (2010a): "Essay on the Origin of Language", en BARNARD, Frederick M. (edit.), J. G. Herder on social and political culture (Cambridge, Cambridge University Press) pp. 115-177.

Herder, Johann Gottfried von (2010b): "Ideas for a Philosophy of the History of Mankind", en Barnard, Frederick M. (ed.), J. G. Herder on social and political culture (Cambridge, Cambridge University Press) pp. 253-326.

Hoeflich, Michael H (1989): "Savigny and his Anglo-American Disciples", American Journal of Comparative Law, vol. $37 \mathrm{~N}^{\circ} 1$ : pp. 17-37.

Kantorowicz, Hermann. (1937): "Savigny and the Historical School of Law", The Law Quarterly Review, vol. $53 \mathrm{~N}^{\circ}$ 3: pp. 326-343.

Kennedy, Duncan (2010): "Savigny's Family/Patrimony Distinction and its Place in the Global Genealogy of Classical Legal Thought", American Journal of Comparative Law, vol. $58 \mathrm{~N}^{\circ}$ 4: pp. 812-842.

KnUdson, James. (2002). "Influence of the German Concepts of Volksgeist and Zietgeist on the Thought and Jurisprudence of Oliver Wendell Holmes", Journal of Transnational Law and Policy, vol. $11 \mathrm{~N}^{\circ}$ 2: pp. 407-419.

Kutner, Luis (1972): "Savigny: German Lawgiver", Marquette Law Review, vol. 55, N²: pp. 280-295.

Mollnau, Karl A. (1989): "The Contributions of Savigny to the Theory of Legislation", American Journal of Comparative Law, vol. 37, $\mathrm{N}^{\circ} 1$ : pp. 81-93. 
LunA-VINUEZA, David Ricardo " "Savigny, Herder y la tensión entre particularismo y universalismo..."

Neme, Marta Lucía (2012): "El error como vicio del "consentimiento" frente a la protección de la confianza en la celebración del contrato", Revista de derecho privado, $\mathrm{N}^{\circ} 22$ : pp. 169-218.

Reimann, Mathias (1989): "The Historical School Against Codification: Savigny, Carter, and the Defeat of the New York Civil Code", American Journal of Comparative Law, vol. 37, $\mathrm{N}^{\circ} 1$ : pp. 95-119.

Reimann, Mathias (1990): "Nineteenth Century German Legal Science", Boston College Law Review, vol. 31, № 4: pp. 837-897.

SAVIGNY, Friedrich Karl von (1949): "Los fundamentos de la ciencia jurídica (\$\$ 4-16, 32 58 del Sistema de derecho romano actual)", en A.A.V.V., La ciencia del derecho (Buenos Aires, Editorial Losada) pp. 29-232.

SAVIGNY, Friedrich Karl von (1977): De la vocación de nuestro siglo para la legislación y la ciencia del derecho (Buenos Aires, Editorial Heliasta).

Savigny, Friedrich Karl von (2004): Metodología jurídica (Buenos Aires, Valleta Ediciones).

Wintgens, Luc J. (2010): "From Law without a Science to Legal Science without the Legislator: the German Historical School and the Foundation of Law", Statute Law Review, vol. $31 \mathrm{~N}^{\circ}$ 2: pp. 85-106.

Zuleta, Enrique (1977): "Savigny y la teoría de la ciencia jurídica", Anuario de Filosofía del Derecho, $\mathrm{N}^{\circ}$ 19: pp. 57-82. 